\title{
EL PROBLEMA DE LA CONFIANZA DESDE LA HERMENÉUTICA FILOSÓFICA: COMPRENDIENDO SUS RENDIMIENTOS INTERPRETATIVOS
}

EN LA SOCIEDAD CONTEMPORÁNEA

\author{
César Marínez Sánchez \\ Universidad de Chile \\ cdmarinez@uchile.cl
}

\begin{abstract}
RESUMEN / ABSTRACT
El objetivo del siguiente artículo es observar los rendimientos de la hermenéutica filosófica para interpretar el problema de la confianza en nuestras relaciones sociales. En primer lugar, se identifican sus fundamentos para comprender a través de esta tradición filosófica su giro lingüístico e importancia para estudiar dicho concepto (I); en segundo lugar, se analiza desde la idea del 'lenguaje' y del 'diálogo' el problema de la confianza en las relaciones contextuales (II). Por último, se reflexiona sobre cómo la racionalidad científica proyecta confianza o desconfianza en un mundo en donde cada vez sus enunciados deben comprometerse con la vida humana (III). Este artículo es relevante pues busca revalorizar a la filosofía para el análisis de un concepto que ha sido estudiado principalmente desde las ciencias sociales. En este sentido nos apoyamos en la hermenéutica filosófica en pos de observar sus posibilidades para conceptualizar y analizar la confianza en la sociedad contemporánea.
\end{abstract}

Palabras clave: confianza, hermenéutica filosófica, lenguaje, ciencia, ética.

\section{THE PROBLEM OF TRUST FROM PHILOSOPHICAL HERMENEUTICS}

The objective of the following article is to observe the performance of philosophical hermeneutics to interpret the problems of trust in our social relations. In the first place, we will identify its foundations to understand through this philosophical tradition its linguistic turns and importance to study the problem of trust (I); Secondly, the problem of trust in contextual relationships will be is analyzed from the idea of 'language' and 'dialogue'(II). Finally, we will focus on how scientific rationality projects trust or distrust in a world where each time its statements must be committed to human life; (III). This article is relevant because it seeks to revalue philosophy for the analysis of a concept that has been studied mainly from the social sciences. In this sense we rely on philosophical hermeneutics in order to observe its possibilities to conceptualize and analyze trust in contemporary society.

KEYWORDS: Philosophical hermeneutics, trust, language, science, ethics. 


\section{Introducción}

$\mathbb{R A}$ La confianza como concepto moderno parece condicionar en la sociedad toda posibilidad de construir una supuesta relación armoniosa en un mundo complejo, colocándose como especie de garantía existencial que les permite a los hombres o a las instituciones involucrarse en contextos de incertidumbre por un futuro que no se conoce cuando uno se relaciona con la otra parte (Dunn 1988; Barber 1983; Luhmann 1996; Rojas 2001; Möllering 2006, 2008; Faulkner 2011). Pero también, la emergencia del fenómeno de la confianza en la sociedad contemporánea se transforma en un problema cuando oímos que persisten situaciones decepcionantes reflejadas en una supuesta crisis institucional o ética (Touraine 1997; Apel 1989; Cortina 2006). Solo basta con escuchar que otros vivencian el desempleo, las guerras, las inequidades, la corrupción, los problemas medioambientales, la pobreza, el no respeto a los derechos sociales, la discriminación por género, raza o nivel socioeconómico, entre otros, para sospechar de lo que se dice sobre el bienestar, la paz, el desarrollo, el progreso, la transparencia, el reconocimiento o la justicia social. En este sentido, la confianza es paradojal, porque si ella es necesaria también es improbable. Le permite a los hombres o a las instituciones confiar o ser dignos de confianza, sabiendo también que las expectativas que se construyen en torno a ellos pueden verse tensionadas al entrar en una relación.

Con esta problemática, mucho se ha dicho sobre cómo es posible 'reconstruir' confianza a partir de principios valóricos como la solidaridad o la justicia (Apel 1989; Coleman 1990; Baynes 1991; Rehg 1997; Chambers 1996; Rawls 1996; Honneth 1996; Fukuyama 1996; Sztompka 1999; Seligman 1997; Habermas 2000; Nooteboom 2002; Uslaner 2002; Cortina 2006; Meehan 2013) o bien, sobre la necesidad de observarla como una especie de inclinación natural en el hombre equivalente a la sinceridad, la simpatía o la honestidad como condición para confiar o desconfiar en el otro (Lewis y Weigert 1985; Baier 1986; Holton 1994; Jones 1996; Nickel 2007). Se intenta preguntarse por cómo revitalizar las relaciones inter-personales o institucionales, en un mundo en donde la generalización de expectativas no es tan obvia como supuestamente se espera. Es decir, la confianza como paradoja se ha tornado en un problema filosófico relevante a investigar para preguntarse por cómo es posible la confianza social.

No obstante, poco se le ha puesto el foco a analizar el problema de la confianza desde un marco interpretativo que ponga entre paréntesis en su análisis sus valoraciones morales, es decir, que pueda hacerse cargo de su paradoja desvistiéndola de una fundamentación moral en las conciencias y colocar el problema en los enunciados que se conocen en el mundo. Frente a esta pretensión, la hermenéutica filosófica parece contener elementos que son claves para lograr esa tarea. Se cree que con esta aproximación uno podría analizar un problema de confianza para reflexionar sobre su paradoja sin reducir la mirada a una certeza apriorística de solidaridad moral (Luhmann 2013).

Con esta aproximación es el lenguaje que como condición originaria estaría configurando la posibilidad de lanzarse al mundo de la confianza, pero también con él, los hombres aprenden a sospechar en el mundo cuando tienen que entrar en una relación social. Como sostiene Alex Honneth (2003), siguiendo a Gadamer, las condiciones existenciales de la experiencia a través del lenguaje tienen la capacidad de poder 
informar, en un presente dado, sobre la posibilidad de algún quiebre en nuestras rutinas justamente por los contenidos referenciales que transitan en nuestro existir fáctico. Dichos elementos darán cuenta de que hay una confianza originaria en el lenguaje, pero sin perder su dimensión reflexiva que la contiene, ya que su historicidad permite que uno pueda estar siempre alerta a una situación de desconfianza. Es decir, la sospecha se hace explícita cuando "la productividad de esta 'auténtica experiencia' nos informa sobre cualquier error de una universalización categorial con la que conocemos al mundo" (Honneth 2003; 8).

El objetivo del siguiente artículo es observar los rendimientos de la hermenéutica filosófica para interpretar el problema de la confianza en nuestras relaciones sociales. En primer lugar, se identifican sus fundamentos para comprender a través de esta tradición filosófica su giro lingüístico e importancia para estudiar dicho concepto (I); en segundo lugar, se analiza desde la idea del 'lenguaje' y del 'diálogo' el problema de la confianza en las relaciones contextuales (II). Por último, se reflexiona sobre cómo la racionalidad científica proyecta confianza o desconfianza en un mundo en donde cada vez sus enunciados deben comprometerse con la vida humana (III). Este artículo es relevante pues busca revalorizar a la filosofía para el análisis de un concepto que ha sido estudiado principalmente desde las ciencias sociales. En este sentido nos apoyamos en la hermenéutica filosófica en pos de observar sus posibilidades para conceptualizar y analizar a la confianza en la sociedad contemporánea.

\section{Giro lingüistico desde la hermenéutica filosófica continental para el estudio de la confianza}

La lógica del pensamiento hermenéutico filosófico se ubica “en el grupo de los saberes llamados emancipatorios que emergen a partir de la crisis de la razón moderna y del agotamiento de la filosofía de la conciencia" (Bentolia 2003). Su tarea estará en tratar aquellos problemas ligados al uso del lenguaje por medio de la explicación de la estructura del comprender humano. Su objetivo será tensionar aquella ansiedad cartesiana (Dummett 1990), que buscaba elevar por encima de todo, a la razón como fórmula fundacionalista de todo conocimiento para ordenar "el caos intelectual y moral en donde todo vale" (Bernstein 1983: 332). Lo que importa ahora es desmantelar este principio explicativo, fragmentarlo y dar cuenta de que el comprender opera antes las proposiciones que se validan en el mundo (Habermas 2011).

En este sentido, se empieza a poner el foco en el lenguaje como una condición necesaria para conocimiento, más allá de ser considerada como un mero instrumento que fundamenta toda verdad a partir de una mera copia con una realidad externa. Es decir, el lenguaje será visto como una "una unidad e igualdad originaria de todo lo espiritual, una unidad originaria de todas las cosas del espíritu" (Schleiermacher en Dilthey 2000: 75) y no como un mero conducto para comunicar descubrimientos (Locke en Lafont 1993). Como una estructura originaria, permitirá englobar a priori todas las formas de desarrollo del espíritu (Coseriu, Pastene \& Katabek 2006), como condición para poder hacer transitar las certezas sensibles hacia lo universal, hacia "el ser en general" 
(Hegel 1992; 65). Englobará, ahora en su interior, la posibilidad de transitar a todas las formas de desarrollo del espíritu y, en consecuencia, él será la determinación de toda posibilidad de ser en el mundo, como condición para confirmar verdades.

Por tal razón, la hermenéutica filosófica se adentra en aquellas corrientes filosóficas que buscan solucionar y darle una salida mucho más humana, que es lo que Dummett (1990) llama el escándalo filosófico, es decir, el supuesto vacío metodológico y sistemático que la filosofía cartesiana debía llenar para descubrir sus verdades absolutas de forma coherente y consistente, desde sus fundamentos últimos (Dummett 1990). Como propuesta, se tendrá entonces la pretensión de superar-o llenar ese vacío metodológico- la "ahistoricidad del comprender" (Heidegger 2012) para poder así interpretar el "sentido inmanente de los propios enunciados a partir de una condición lingüística” (Ricoeur 1997, 2012). Se necesitará de una consistencia teórica y metodológica para lograr alcanzar ese "comprender originario" que permita justificar a las verdades desde un "campo a-temático de lo posible que precede a toda comprensión [que] está esencialmente por la auto-trasmisión de la existencia" (Heidegger en Rodríguez 2010: 38) en cuyo común denominador será, como lo hemos venido reiterando, el lenguaje:

El común denominador de dicho giro lingüístico lo constituye, indudablemente, la crítica a la concepción tradicional del lenguaje como un instrumento para la designación de entidades independientes del lenguaje o para la comunicación de pensamientos pre-lingüísticos. Solo tras la superación de esa comprensión del lenguaje, es decir, tras reconocer que al lenguaje le corresponde un papel constitutivo en nuestra relación con el mundo, puede hablarse en sentido estricto de un cambio de paradigma de la filosofía de la conciencia a la filosofía del lenguaje (Lafont 1993: 15).

Si para la filosofía de la conciencia su foco se encontraba en descubrir verdades a través de la razón del cogito que piensa, para la hermenéutica filosófica, la razón es el lenguaje, ya que "sin la palabra no hay ni razón, ni mundo" (Hamann en Lafont 1993: 23). Con esta nueva argumentación, las referencias sobre el mundo estarían perteneciendo al conjunto de una existencia histórica y simultánea con todo lo que pueda estar presente en ella (Gadamer 2012: 483) y por lo tanto, implica que "no haya propiamente renuncia a la razón, sino un descubrimiento de su condicionalidad lingüística: la razón no está más allá del lenguaje, sino que, al igual que el propio ser, es lenguaje" (Arrizabalaga 2005: 248). La razón como lenguaje (Lafont 1993) implica, en este sentido, que se tenga como foco a la interpretación como forma de desplazamiento de la reflexión, desde el ser hasta el lenguaje (Arrizabalaga 2005) y por lo tanto, ella viene a ser una especie de base estructurante y estructuradora de nuestra relación con el mundo (Rodríguez 2010). Solo de esa manera se estaría tomando en cuenta al ser mismo en su haberse revelado, como lo abarcante del ser mismo (Jasper 2012: 466).

Con estos pilares emancipatorios, se parte entonces de la idea de que hay condicionalidad lingüística que justifica todo tipo de verdades, más allá de una realidad ahistórica e independiente de las condiciones existenciales de vida humana (Gadamer 2010). Este giro lingüístico tiene sus implicancias para poder observar, de otra manera, el problema de la confianza, pues ahora su abordaje no dependería de categorías 
apriorísticas de la conciencia. El lenguaje se constituirá como aquel principio explicativo que fundamenta todo conocimiento valido. En consecuencia, para poder entender un problema de confianza en nuestros actos de habla se debe tomar al lenguaje como un elemento originario y pre-teórico que garantiza apriorísticamente las certezas que se construyen en el mundo para toda creencia posible.

\section{La importancia del lenguaje en el diálogo para la construcción de confianza}

Si nos posicionamos en la tesis antes planeada, en la cual el lenguaje, como razón, es el que justifica la creencia en una 'confianza primera', frente a lo que vivenciamos en nuestra cotidianeidad, entonces también debemos tener en cuenta que el lenguaje, como razón, encuentra "su ser verdadero en la conversación" (Gadamer 2012b: 203). Esto significa que querer expresar con verdad algo a alguien, en una conversación, implica que esta última posea su propia temporalidad e historicidad (Gadamer 2012b). De lo contrario, no tendríamos la posibilidad de confiar o desconfiar en la intencionalidad del otro, ya que no tendríamos la posibilidad de interpretar -no tendríamos pistas suficientes para poder aceptar o rechazar su oferta de lo que hemos oído anteriormente sobre alguien o algo. Solo de esa manera, a través de la conversación, podremos comprender el misterio de la interioridad del otro (Gadamer 1997: 129), es decir, comprenderemos las razones de quien habla para así poder ofrecerle una respuesta a sus preguntas:

El arte de comprender consiste, seguramente y ante todo, en el arte de escuchar. Sin embargo, a ello hay que añadir la posibilidad de que el otro pueda tener razón. El otro de entrada se encuentra en una mala situación si ambos lados no sienten eso [...] Siempre que se quiera comprender a otro o una cosa, debemos preguntarnos cuál será la pregunta respecto a la cual esta o aquella manifestación lingüística constituirá la respuesta. Hasta que no llego a este punto, no he comprendido absolutamente nada (Gadamer 2012: 227-8).

Si el lenguaje siempre tiene historia (Heidegger 2012) es porque en un presente dado, escuchamos al otro en una conversación, teniendo en cuenta que el "oír abarca todo el universo de lo pensante como aquel universo de las lenguas" (Gadamer 2002: 68). En consecuencia, para poder comprender, se tiene que aprender a escuchar. Pero este escuchar es posible porque el existir mismo que refiere al mundo guarda su ser ahí en la conversación:

Cuando usamos la palabra, ésta interpela siempre a alguien, pero dice además siempre otra cosa, aquello a lo que nos referimos. Junto a este hecho de que el nombre es una interpelación en ambas direcciones, se da un primer paso que conduce evidentemente a una presentación de esta clase, vigilia a la que hace referencia el ahí (Gadamer 2002: 70).

El hacer referencia 'al ahi', implica poder entender a la interioridad del otro, significa poder "ir a lo que se dice, aunque haya asentimiento" (Gadamer 2002: 73). Por eso, 
la confianza así como la desconfianza hacia lo que se dice, en nuestras relaciones contextuales, pueden transformarse; pero siempre haciendo referencia de forma latente 'al ahi', cuando conversamos con el otro:

El hablar no es hacer deducciones lógicas es, en cierto sentido, dominar la palabra y ello produce algo que se ha de interpretar por el contexto en el sentido más fuerte de éste. Porque el contexto no son aquí solo las palabras, es el contexto de la vida entera (Gadamer 1997: 134).

La vida entera -el ahí, el ahora-, configura toda posibilidad de poder confiar o desconfiar en una conversación, entre quien escucha y quien quiere dar a conocer algo a alguien. Se confía 'primeramente' en un horizonte histórico de las cosas; la vida entera que se proyecta en la palabra que se escucha o que hemos venido oyendo en nuestras vivencias. Por tal razón, "la palabra hablada no es mía, ha quedado entregada al oír de otros. En eso consiste la gran responsabilidad del hablar: en el que la palabra una vez dicha, no puede ser retirada. La palabra hablada pertenece al que oye" (Gadamer 2002: 70). En otras palabras, que no confiamos en el otro por lo que éste dice, sino más bien por lo que ha dicho sobre ese algo o alguien. Solo de esta manera resulta posible confiar en mi otro yo; cuando logramos interpretar algún enunciado referente al mundo sobre lo que hemos oído sobre ese algo a través de lo que Heidegger conceptualiza como 'el hablilla', que

[s]e experimenta de modo justamente no expreso, no actual; es como un existir por el cual todo es vivido. Precisamente porque constituye la publicidad y, en cuanto tal, la normalidad, que está ahí y que cualquiera puede entender fácilmente, nada de lo que de lo que pasa se escapa. El hablilla habla de todo con una peculiar falta de sensibilidad para con las diferencias. En cuanto tal normalidad, el ahora seguro, ahora en cuanto lo que siempre, la publicidad es el modo de ser del 'uno': uno dice, uno oye, uno cuenta, uno supone, uno espera, uno está a favor de que...El hablilla no es de nadie, nadie se responsabiliza de haberla dicho (Heidegger 2013: 52).

El hablilla es el hablar mismo sobre lo que hemos oído -sobre las cosas del mundo-que se actualiza en una práctica contextual, en forma de conversación. Es lo que Gadamer define como aquella palabra hablada que no es mía sino que ha sido entregada al oír de otro en el mundo a partir del diálogo.

La función del diálogo es que el decir o afirmar algo implique una relación provocativa con el otro, que provoque una respuesta, y que la respuesta suministre la interpretación de la interpretación del otro (Gadamer 1997: 134).

Solo de esta manera podremos tener las pistas suficientes para poder confiar o desconfiar en mi otro yo, puesto que se nos hacen familiares. Permite que podamos confiar en lo que hemos escuchado alguna vez en nuestras vidas sobre el mundo, para confiar en el otro, ya que es aquel el fantasma antes mencionado que, de forma latente, transita por sobre nuestras vivencias a través del lenguaje. Con ello es posible creer o no creer en el conocimiento que nos quiere transmitir mi otro yo; nos permite saber en la intencionalidad 
del otro si el conocimiento que nos quiere dar a conocer es efectivamente verdadero en un contexto dado.

En síntesis, con el lenguaje sabemos que podemos confiar o sospechar de las afirmaciones del hablante, pues haciendo uso del lenguaje como medio originario y existencial, podremos entender lo que emite el hablante, precisamente porque conocemos el contenido existencial de su afirmación. Dicho contenido depende del lenguaje como condición para entender al otro y creer en sus afirmaciones cuando se refiere al mundo (Habermas 2011). Hay confianza hacia los enunciados que escuchamos en el mundo porque se sabe de antemano que el lenguaje le ha dado un sentido temporal para evaluar o sospechar de ellos en una relación. Lo ya interpretado nos da cuenta de un saber que transita y que se encuentra de forma implícita en nuestros actos para hacerse entender o para entender al otro. Esto nos confirma, entonces, la necesidad de una familiaridad de lo que conocemos para abrirnos al mundo y así confiar en el otro a través de la comprensión; para poder saber si mi otro yo interpreta mi oferta de confianza de la misma forma que yo en la conversación, porque ambos sabemos que nos estamos refiriendo al mismo objeto en un contexto dado en un mundo familiar (Luhmann 1988).

\section{Desplazamiento hacia un modelo comprensivo de racionalidad científica para la construcción de confianza social}

Si para la hermenéutica filosófica la razón es el lenguaje, entonces podríamos decir que en ella existe una especie de 'confianza primera' que se antepone a toda posibilidad de confiar o desconfiar en nuestras relaciones intersubjetivas o en la praxis cuando hablamos de verdades sobre el mundo. El lenguaje predetermina todo lo contingente pero, a su vez, confiar en él no se valida por representar correctamente una realidad a-histórica e independiente. Estaríamos volviendo, de nuevo, a aquel mundo racional e idealista en el cual el cogito que piensa, a través de la razón, confía en sí mismo y se compromete a descubrir verdades que son supuestamente eternas, excluyendo todo aquel conocimiento que proviene del lenguaje no formal y contingente. Solo se haría uso del lenguaje como un mero instrumento tecnificado que fundamenta todo conocimiento únicamente desde un yo pensante, que tiene la certeza en la razón por sobre todo tipo de enunciación que justifica verdades contingentes o cambiantes.

La hermenéutica filosófica recupera, en este sentido, la necesidad de creer en verdades que son contingentes, pero a su vez, dependen del existir mismo a través del lenguaje. Es decir, "Deben ponerse a la vista en lo ya interpretado y público la actualidad de determinadas categorías del existir, para los cuales se trata de estar despierto. Ontológicamente la actualidad supone: el presente del ahora, el uno, el estar con los demás, con los otros; nuestro tiempo" (Heidegger 2013: 50).

Confiar o desconfiar depende entonces de una 'confianza primera'; la confianza en el lenguaje ontologizado, como condición constitutiva de todo conocimiento sobre el mundo que trasciende todo tipo de justificación contextual. Para confiar en las verdades sobre el mundo se requiere entonces de un fantasma que se echa a andar en 
el existir fáctico (Heidegger 2013), actualizándose a cada instante en nuestras prácticas cotidianas cuando me relaciono con mi otro yo para poder hablar de verdades. Solo de esta manera podemos movernos de un punto a otro en el mundo que conocemos, porque existen condiciones preestablecidas que permiten arrojamos a circunstancias que se nos hacen familiares, para poder confiar o desconfiar; son condiciones que por las cuales sería imposible interpretar cualquier enunciado que refiere a nuestro mundo fáctico;

Todo enunciado tiene su horizonte situacional y su función interpelativa es solo la base para su conclusión ulterior de que la historicidad de todos los enunciados radica en la finitud fundamental de nuestro ser. Que un enunciado es más que una simple actualización de un fenómeno presente significa, ante todo, que pertenece al conjunto de una existencia histórica y es simultáneo con todo lo que pueda estar presente en ella (Gadamer 2012a: 483).

Lo anterior implica que la confianza hacia las verdades en nuestras relaciones finitas pueda transformarse también en desconfianza, cuando "la pretensión de verdad se convierte en un medio de lucha también por lo no verdadero" (Jasper 2012: 462). Lo no verdadero puede abrirse al mundo como una nueva actualización de desconfianza hacia lo que, en un momento temporal, fue considerado como verdadero, precisamente porque conociendo al mundo, se aprende de la "productividad de esta 'auténtica experiencia', la cual nos informa sobre cualquier error de una universalización categorial con la que conocemos al mundo" (Honneth 2003: 8). Por eso, la verdad y la no verdad están juntos; la finitud de las manifestaciones del ser corresponde a la finitud del 'Dasein' humano (Heidegger 1979, 2012).

Si el lenguaje como razón es el que nos orienta en la conversación, para comprender la intencionalidad del otro y así saber si confiar o desconfiar en nuestros contextos, no solo por lo que mi otro yo dice, sino también por lo que dice la palabra, entonces cabe preguntarnos ahora por cómo la palabra cientifica puede hacernos creer que sus enunciados son verdaderos, teniendo en cuenta que para la hermenéutica filosófica, "el fenómeno de la comprensión atraviesa todas las referencias humanas del mundo" (Gadamer 2012). También vale ahora preguntarnos por cuáles son las consecuencias de que la ciencia quiera hacernos creer en verdades sobre el mundo que son irrefutables, teniendo en cuenta que el gran problema de ella, es "haber triunfado en su ilusión de querer renovar al platonismo en su renuncia a lograr el ser que es" (Blumenberg 2004: 36). En otras palabras, si la ciencia experimental tendrá la pretensión de descubrir aquellas verdades sobre aquel mundo externo, como copia fiel de la realidad, dejando bien guardado sus orígenes o el pasado que los determina, entonces aquella estaría olvidando al ser que es que equivale al lenguaje como razón constitutiva de todo conocimiento humano.

Lo anterior lo podemos ver reflejado con la llegada de la Edad Moderna, cuando en dicho periodo aparece un nuevo concepto de ciencia que, bajo su propia lógica, logra emanciparse del lenguaje y desarrolla en su interior sus propios sistemas de designación y de representación simbólica (Gadamer 2012: 32). Lo anterior implica que las expectativas de la racionalidad científica están en "la seguridad de emplear mi razón en todo e irse acostumbrando a concebir los objetos con mayor claridad y 
distinción" (Descartes 2006: 55). Por lo tanto, la voluntad del saber queda reducida a una "autoconciencia que apela a una razón, inventada por ella misma" (Heidegger en Constante 2004: 11) y en consecuencia, esta invención de la ciencia hace que "la experiencia de la verdad queda, por así decir, controlada por la metodología científica" (Gadamer 2012). Es decir, el pensar o la voluntad de saber se tecnifica y la enunciación de verdades sobre el mundo quedan garantizadas por sus descubrimientos, "como aplicación de hechos que no son más que determinaciones reducidas a elementos puramente lógicos" (Horkheimer 2004: 228).

Esta formalidad, para la hermenéutica filosófica, implica que la teoría se limite a formular los resultados de los experimentos para fundamentar todo conocimiento sobre el mundo de lo que realmente pasa (Gadamer 2012), dejando de lado la esencia históricamente acontecida de la verdad (Pöggeler 1986) en la elaboración de sus datos. En otras palabras, las verdades pierden su temporalidad; pierden, por así decir, su horizonte existencial que le permite al investigador saber implicarse en lo comprendido para aportar en el propio presente, al horizonte del pasado (Rodríguez 2010).

Estas breves argumentaciones, anteriormente mencionadas, tienen implicancias de gran envergadura para la vida humana, en su conexión con el problema de la confianza en las verdades sobre el mundo. En primer lugar, los saberes sin historia no nos darían la posibilidad de poder aprender a saber sospechar de aquellas verdades que pretenden ser absolutas e irrefutables. El pensar epistémico sería, por así decir, aquella autoridad suprema que nos garantizaría toda validez absoluta y, por lo tanto, nos haría creer firmemente en una confianza primera en la racionalidad científica. Existiría una especie de conformismo, fe o esperanza hacia aquella fuerza lógica que logra persuadirnos (Gadamer 1997) sobre cuáles son los enunciados que pueden considerarse como válidos en el mundo, sin posibilidad de dialogar para reflexionar o sospechar en dichas enunciaciones. Si "el diálogo se basa en una estructura previa pre-lingüística que nos permite entender o ir a lo que se dice" (Gadamer 2002: 73), pues con la ciencia no habría posibilidad de asentimiento, puesto que el oír con el otro está determinado por lo que dice la palabra de la ciencia. En otras palabras, estaríamos todos predeterminados a no poder reflexionar o a no tener una conciencia crítica de lo que pasa en el mundo o sobre lo que pasa realmente en él, ya que el saber epistémico es quien tiene el poder en darnos a conocer al mundo más real.

En segundo lugar, si el pensar deja de pensar al ser (Heidegger 1979) por el simple hecho de validar hechos que son irrefutables por un lenguaje propio de representaciones simbólicas que se independiza de aquel lenguaje originario y preteórico de la vida humana, pues la experiencia en la verdad dejaría de ser contingente por el simple hecho de ocultar la posibilidad de creer en otras verdades que vivenciamos en el mundo de la vida, cuando nos relacionamos con mi otro yo a través del lenguaje. "Pues la comprensión de nuestro mundo sedimentada en el leguaje no puede ser absorbida en su totalidad por la posibilidades de conocimiento específicas de la ciencia" (Gadamer 2012: 31). Sin la posibilidad de diversificar las verdades no habría posibilidad de poder aceptar, rechazar o confiar en otras referencias humanas sobre el mundo, ya que el lenguaje científico totalizaría, desde sus representaciones simbólicas, las verdades cuando conversamos sobre 'las cosas'. 
En consecuencia, se limita la contingencia limitando "ser para la existencia humana que es siempre ocuparse de las propias posibilidades del ser [...] se constituyen como tales en el seno de un estar ya en situación" (Rodríguez 2010: 35). Se limita a la contingencia que se manifiesta, precisamente, por la totalidad de referencias humanas del mundo y que, a través del ser, nos permite arrojarnos hacia delante para saber aceptar la oferta de que mi otro yo -como por ejemplo, la ciencia-cumpla con mis expectativas.

\section{Conclusión}

Para la hermenéutica filosófica la construcción de expectativas es posible justamente a través del lenguaje. Dependerá, en otras palabras, de la familiaridad que los hombres han venido conociendo a través de los contenidos referenciales que se reproducen sobre la existencia humana -para comprender en lo comprendido- y esto es, por así decir, condición de los hombres para poder abrirse a la pluralidad de mundos contingentes en sus prácticas contextuales. El lenguaje se coloca entonces como un componente preteórico, originario de la vida humana y existencial que se actualiza en cada presente para la construcción de expectativas -o para alertar sobre un posible quiebre en sus rutinas o hábitos en sus vidas cotidianas.

Es a través del lenguaje que los hombres pueden aprender a interpretar o comprender al otro cuando conversan entre sí; sin él sería imposible tener las condiciones para aprender a escuchar al otro y otorgarle algún tipo reconocimiento a sus afirmaciones sobre el mundo. En este sentido, cuando confío o desconfío es porque comprendemos al otro a través del lenguaje, quien abre las condiciones para saber si lo que se enuncia como supuestamente verdadero se acepta o se sospecha en la conversación. El lenguaje abre las condiciones para aceptar las expectativas de los otros, compartir creencias o dudar de ellas en un mundo que es contingente, pero a su vez, esta contingencia es paradojal pues existiría por así decir una confianza primera hacia una trama de significados que se han venido construyendo a lo largo de la historia de la humanidad a través del lenguaje.

En relación con la ciencia que produce enunciados empíricos, ésta deberá también comprometerse con la vida humana; deberá conectarla y reconocer en sus enunciados un interés práctico para los hombres. La preocupación intelectual de esta corriente está precisamente en colocar al saber especializado en su lugar que corresponde y dar también cuenta de "la función reveladora del mundo del lenguaje ordinario" (Habermas 2011: 70). A la hermenéutica filosófica le interesa dar cuenta de que el lenguaje no le pertenece únicamente a la ciencia; el lenguaje deja de ser un instrumento tecnificado por la ciencia para enfatizar el hecho de que aquel le pertenece a la vida humana, con la cual se construye una la pluralidad de formas de sentido para referirse al mundo diversamente.

Si vinculamos esto último con el problema de la confianza, los hombres no estarán sujetos a confiar únicamente en los enunciados que sostiene la ciencia. Más bien, se debe reconsiderar las verdades que se construyen en el mundo ordinario y que son también válidas para reconocer otras verdades contextuales. La confianza no está en una confianza primera en la racionalidad científica, sino, como lo mencionamos en 
el párrafo anterior, en el lenguaje como componente preteórico existencial de la vida humana; es la confianza primera en la condición originaria del lenguaje la que permite, por así decir, la confianza reflexiva en la conversación o en el diálogo.

Con este análisis conceptual desde la hermenéutica filosófica, lo que se despliega es la necesidad de observar la confianza como un proceso contingente y dinámico condicionado por el lenguaje, ya que lo 'ya producido' que este produce, siempre se actualiza en un presente, para seguir decidiendo -o estar siempre alertas- si entrar o no en una relación de la confianza', en el entendido de que la confianza está siempre abierta a todo tipo de posibilidades desde diferentes posiciones particulares que se actualizan a cada instante (Luhmann 1988,1996). En tal sentido, la emergencia de la confianza no se agota ahí donde hubo decepción, así como tampoco se acaba porque en algún momento de nuestro horizonte temporal de nuestra experiencia alguien cumplió con nuestras expectativas. De ser así solo tendríamos un único pase de entrada que ya habríamos agotado desde hace mucho tiempo. En este último punto, la desconfianza cobra relevancia, pues también nos abre las condiciones para lanzarnos de nuevo al mundo -habiendo aprendido de las decepciones- para confiar de otra manera a través del lenguaje.

Lo anterior nos hace ver entonces que la confianza absorbe la incertidumbre del mundo y la transforma en contingencia - una vez que hemos entrado en el mundo de la confianza con la ayuda del lenguaje. Sus rendimientos están justamente en identificar en la confianza sus procesos, a través del lenguaje, las que posibilitan siempre nuevos escenarios para seguir confiando o para confiar de otra manera -cuando en algún momento hemos sufrido alguna decepción. Como proceso, la confianza se construye entonces en el tiempo, teniendo siempre la posibilidad de construir expectativas de persistencias - o de reforzarlas en caso de haber sido defraudado en algún momento en este camino (Barber 1983). Como proceso, su forma de garantizarnos estabilidad es entonces dinámica, ya que una vez encaminados, se abrirán siempre nuevos escenarios para poder seguir confiando, frente a posibilidad de volver a la incertidumbre. Pero a su vez, esta incertidumbre gatillará de nuevo la posibilidad de lanzarnos al mundo, pues sabemos - con lo ya interpretado-que tarde o temprano tendremos que volver a confiar.

\section{Referencias bibliográficas}

Acero, J. J., E. Bustos \& D. Quesada (1989), Introducción a la Filosofía del lenguaje. Madrid: Cádetra.

Apel, K. O. (1989), "La situación del hombre como problema ético", en X. Palacios y F. Jarauta, eds., Razón, Ética y Política: El conflicto de las Sociedades Modernas. DF: Anthropos.

Arrizabalaga, L. J. G. (2005), "Hermenéutica del lenguaje y simbolismo". Endoxa: Series Filosóficas 20: 245-262.

Baier, A. (1986), “Trust and antitrust”, Ethics 96 (2): 231-260. 
Barber, B. (1983), The Logic and Limits of Trust. New Jersey: Rutgers University Press.

Baynes, K. (1991). The normative grounds of social criticism: Kant, Rawls, and Habermas. Nueva York: University of New York Press.

Bernstein, R. (1983), "What is the difference that Makes a Difference? Gadamer, Habermas and Rorty", Philosophy of Science Association 2: 331-359.

Bentolila, H. R. (2003), ¿Qué significa Filosofía del lenguaje? Un esquema de interpretación de la filosofía a partir del giro lingüistico. Universidad Nacional del Nordeste. Argentina. Disponible en: http://www.unne.edu.ar/unnevieja/ Web/cyt/cyt/2003/comunicaciones/02-Humanisticas/H-016.pdf

Blumenberg, H. (2004), Salidas de cavernas. Madrid: La Balsa de la Medusa.

Chambers, S. (1996), Reasonable democracy: Jürgen Habermas and the politics of discourse (Vol. 11). Ithaca NY: Cornell University Press.

Coleman, J. (1990), Foundations of Social Theory. New York: Harvad University Press.

Constante, A. (2004), Martín Heidegger en el camino del pensar. DF: Seminarios. Universidad Nacional Autónoma de México.

Cortina, A. (2006), Ética sin Moral. Madrid: Tecnos.

Coseriu, E., F. Pastene y J. Katabek (2006), "Diez tesis a propósito de la esencia del lenguaje y del significado", Literatura y Linguistica 17: 327-331.

Descartes, R. (2006), Discurso del Método. Madrid: Espasa Calpe.

Dummett, M. (1990), Verdad y otros enigmas. DF: Fondo de Cultura Económica

Dunn, J. (1988), “Trust and Political Agency, in Gambetta", Diego Gambetta (ed). Trust: Making and Breaking Cooperative Relations.

Dilthey, W. (2000), El surgimiento de la hermenéutica, en W. Dilthey, Dos escritos sobre hermenéutica. Madrid: Istmo.

Faulkner, P. (2011), Knowledge on trust. Oxford: Oxford University Press.

Fukuyama, F. (1996). La confianza: las virtudes sociales y la capacidad de generar prosperidad. Buenos Aires: Editorial Atlántida.

Gadamer, H. G. (1996), Verdad y Método. Fundamentos de una hermenéutica filosófica. Salamanca: Sígueme.

(1997), "La hermenéutica de la sospecha", en G. Aranzueque, ed., Horizontes del relato. Lecturas y conversaciones con Paul Ricoeur. Departamento de Filosofía de la Facultad de Filosofía y Letras de la Universidad Autónoma de Madrid.

(2002), Acotaciones hermenéuticas. Madrid: Trotta.

(2012a), ¿Qué es la verdad? (1957), en M. J. Sanz y J. A. Nicolás, eds., Teorías de la verdad en el siglo XX. Madrid: Tecnos.

(2012b), El giro hermenéutico. Madrid: Cátedra.

Garagalza, L. (2005), "Hermenéutica del lenguaje y simbolismo". Endoxa: Series filosóficas 20: 254-261. 
Habermas, J. (2000). Aclaraciones a la Ética del Discurso. Madrid: Trotta. (2011), Verdad y Justificación. Madrid: Ed. Trotta.

Hegel, G. W. F. (1992), Fenomenología del espíritu. DF: Fondo de Cultura Económica. Heidegger, M. (1979), Ser y tiempo. DF: Fondo de Cultura Económica.

(2012), De la esencia de la verdad (1943), en M. J. y J. A. Sanz Nicolás, Teorías de la verdad en el siglo XX. Madrid: Tecnos.

(2013), Ontología: Hermenéutica de la facticidad. Madrid: Alianza.

Holton, R. (1994), "Deciding to trust. Coming to Believe". Australian Journal of Philosophy 72: 63-76.

Honneth, A. (1996), "Patterns of intersubjective recognition: Love, rights, and solidarity", en A. Honneth, The struggle for recognition: The moral grammar of social conflicts, Cambridge: MIT Press.

(2003), "On the destructive power of the third. Gadamer and Heidegger's doctrine of intersubjetivity". Philosophy and Social Criticism 29 (1): 5-21.

Jones, K. (1996), “Trust as an Affective Attitude”, Ethics 107 (1 ): 4-25.

Jasper, K. (2012), “De la verdad” (1947), en M. J. Sanz y J. A. Nicolás, Teorías de la verdad en el siglo $X X$. Madrid: Tecnos.

Horkheimer, M. (2004), Teoría Crítica. Buenos Aires: Amorrortu.

Lafont, C. (1997), "Universalismo y pluralismo en la ética del discurso", Isegoria 17: 37-58.

(1993), La Razón como lenguaje. Una revisión del "giro lingüístico" en la filosofia del lenguaje alemana. Madrid: Visor.

(2002), "Realismo y constructivismo en la Teoría Moral kantiana: el ejemplo de la Ética del Discurso", Isegoria 27: 115-129.

Lewis D. Weigert A (1985), "Social Atomism, Holism and Trust", The Sociological Quartely 26 (4): 455-471.

Luhmann, N. (1996), Confianza. México DF: Anthropos.

(1988), "Familiarity, Confidence, Trust: Problems and Alternatives", en D. Gambetta, ed., Trust: Making and Breaking Cooperative Relations (94108). Oxford: Basil Blackwell.

(2013), La moral de la sociedad. Madrid: Trotta.

Mascareño A., R. Cordero y D. Chernillo (2016), On the Reflexivity of Crisis: Lessons from Critical Theory and Systems Theory. European Journal of Social.

Meehan, J. (2013), Feminists Read Habermas (RLE Feminist Theory): Gendering the Subject of Discourse. Nueva York: Routledge.

Misztal B. (1996), Trust in modern society: The Search for the Bases of Social Order. New Jersey: Wiley.

Möllering G. (2006), Trust: Reason, Routines and Reflexivity. Amsterdam: Elsevier. 
(2008), Inviting or Avoiding Deception through Trust? Conceptual Exploration of an Ambivalent Relationship. Köln: Max-Planck-Institut für Gesellschaftsforschung; working paper.

Nickel, J. (2007), "Trust and obligation-ascription", Ethical Theory and Moral Practice 10: 309-319.

Nooteboom, B. (2002), Trust: Forms, Foundations, Functions, Failures and Figures. UK: Edward Elgar Publishing Limited.

Pöggeler O. (1986), El camino del pensar de Martin Heidegger. Madrid: Alianza.

Rawls, J. (1996), El liberalismo Político. Barcelona: Crítica.

Rehg, W. (1997), Insight and Solidarity: a study in the discourse ethics of Jürgen Habermas. California: University of California Press.

Ricoeur, P. (1997), “Fenomenología y hermenéutica. Cuaderno gris 2: 25-48.

(2012), "Hacia el concepto de "verdad metafórica" (1975), en M. J. Sanz y J. A. Nicolás (2012), Teorías de la verdad en el siglo XX. Madrid: Tecnos.

Rodríguez R. (2010), Hermenéutica y subjetividad. Madrid: Trotta.

Rojas, P. (2001), Confianza y existencia: claves para una interpretación de la filosofía afirmativa del siglo XXI. Universidad de Castilla La Mancha (tesis doctoral). España.

Seligman A (1997), The problem of trust. Princeton: Princeton University Press.

Sztompka P. (1999), Trust: A Sociological Theory. Cambridge: Cambridge University Press.

Touraine, A. (1997), Podremos vivir juntos: iguales y diferentes. México, DF: Fondo de Cultura Económica.

Uslaner, E. (2002), The Moral Foundation of Trust. Maryland: University of Maryland.

Weinsheimer J. (1985), Gadamer's Hermeneutics A Reading of Truth and Method. Yale: Yale University Press. 